\section{Home Made Silicone Rubber Embedding Molds}

Wolfgang Muss

Institute for Pathological Anatomy

Salzburg General Hospital, Salzburg, Austria

Silicone rubber embedding molds may be inexpensive In North America, but are not necessarily so elsewhere in the world. Further, commercial molds are available only in standard sizes and only with standard sizes and shapes of cavities for specimen embedding. If, however, one needs to embed specimens of nonstandard size or shape, wants more embedding cavities per mold than usually available, or is working where commercial molds are expensive or not easily available, then all is not lost. Embedding molds for specimen blocks can easily be made in the lab from silicone rubber. Further, making embedding blocks allows the use of different silicone rubbers which would be of value with unusual embedding resins, or when embedding must be done in unusual environments (such as extreme cold or heat).

\section{The Primary Mold}

Making embedding molds first requires constructing a "negative mold" for casting the silicone to make the final mold. This primary (first) mold can be constructed of Plexiglas or wood covered with a very smooth surface such as melamine or PVC plastic. "Wood" made of compressed and glued wood chips is not recommended for this first mold. PVC or hard PVC (Acryinitrile-styrole-AcryInitrile), or resins like Duroplast, Durovit, etc., can also be used for this, but for this note, it is assumed that Plexiglas is used,

The primary mold is sized according to how large the embedding (final) mold is to be, allowing for any size change during curing, so that the mold will fit into any oven used during embedding. This dimension is important!

To construct the primary mold, glue three Plexiglas plates as sides to a base plate. The fourth side will remain free. Add reinforcement to the base and three side plates joints by gluing strips along the outside of the joints.

As the slide-in plate must fit with precision in this three sided box, the three side plates and the baseplate must not have glue on or at the inner sides or edges. Such would interfere with the exact horizontal plane (of the slide-in plate) needed when curing the silicon rubber mass, as well as with the proper and tight fitting of the slide-in-plate with respect to base-plate and side plates when pouring the silicone mass. The surface of the Plexiglass parts should be thoroughly cleaned (fat free) before gluing by wiping with alcohol (e.g., 100\% EtOH). Rather than using "regular" glue, use chloroform mixed with Plexiglas "sawdust" or commercially available plexiglass glue to glue the sides. This will dissolve the Plexiglas and form a "weld", rather than a simple glue joint. "Regular" glue might result in excess glue coming out of the joint, and will give messy edges to the final product (including in the embedding cavities, see below). Gluing Is facilitated by using a disposable

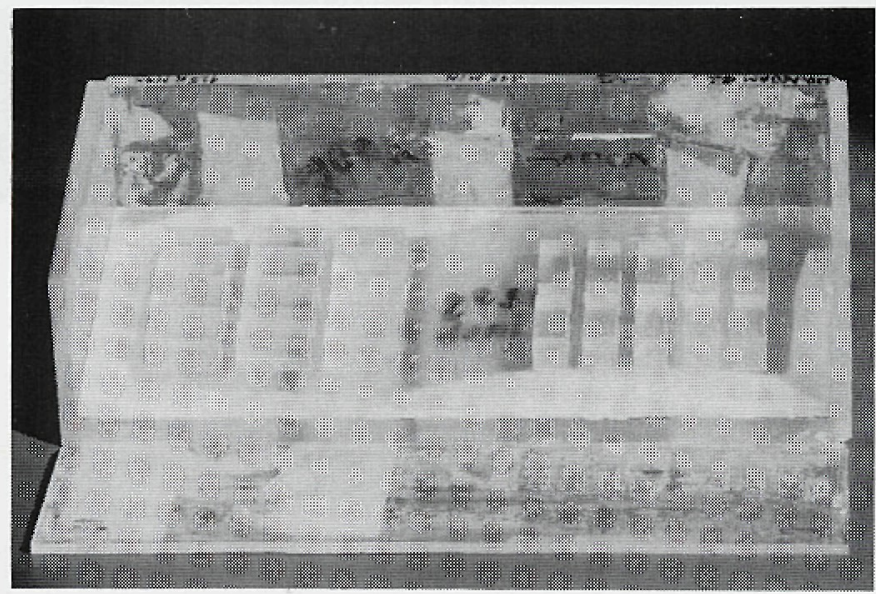

Figure 1A: Primary mold with slide-in plate for making rectangular embedding molds in place. The size of the slide-in plate is $14.8 \times 7.3 \times 0.5 \mathrm{~cm}$. syringe to apply the glue.

The fourth side is left free, so that a slide-in plate with the forms for the embedding cavities can be placed in the primary mold. After placing the slide-in plate in the mold, this fourth side is held to the mold with clamps and/or with strong self adhesive insulation tape. It is a good idea to wrap the ends with plumber's Teflon tape.

The embedding cavities are made by gluing forms to the slide-in plate. These forms are sized and shaped according to the embedding cavities required. Make the forms about $4 \%$ larger than needed to allow for shrinkage during the curing of the silicone mass and/or the embedding resin during polymerization. The forms may be made from any of several different materials, such as metal, Plexiglas, BEEM capsules filled with polymerized resin, and so forth. Be sure to remove the BEEM capsules from the polymerized resin before gluing the resin blanks to the primary mold base-plate. Use as many forms as desired and make as many embedding cavities as needed (see figure 2).

Make identification numbers for the cavities by engraving numbers or letters in normal writing on the tops of the cavity forms. This results in reversed writing on the embedding mold, and in normal writing on the final resin casts. Identification marks can be inscribed in reversed writing on the primary mold or cavity forms so they can be read on the embedding mold.

The primary mold is then assembled: the slide-in plate with cavity forms is slid into the primary mold and the fourth side is then held by clamps or tape.

\section{Casting the Specimen-Embedding Mold}

The embedding mold is cast from silicone rubber. I have found that Elastosil RTV-2 M 4503 with T 35 hardener (Wacker Silicone Co.) at $5 \%$ (wt:wt) works well, but other types will work also. Local availability will determine what is used.

The silicone chosen should have these properties:

$\rightarrow$ Low to medium viscosity, so it is easily pourable, with a pot-life of at least 15-20 minutes;

$\rightarrow$ Moderately "hard", but flexible for easy removal of specimen blocks:

$\rightarrow$ Light or pale in color to aid in orienting specimens during embedding

(Transparent is also good, then a color background can be used);

$\rightarrow$ Should withstand temperatures of 100 to $140^{\circ} \mathrm{C}$ for rapid curing of resin and "tempering" the molds. Tempering emulates aging of the silicone and increases its stability and life. The silicone and conditions will vary if the specimens are to be embedded at unusual temperatures.

With the Elastosil mentioned above, and the resin polymerized at $65^{\circ} \mathrm{C}$, specimens can be embedded, polymerized, and removed by flexing the mold 50 or more times. Polymerizing at $90^{\circ}$ to $100^{\circ} \mathrm{C}$ will shorten the life of the mold.

Follow the manufacturer's specifications for the silicone rubber and hardener/catalyst that you use. Mix in a container (glass or polyethylene) with two to three times the volume of the silicone solution you will be making.

\section{Continued on page 22}

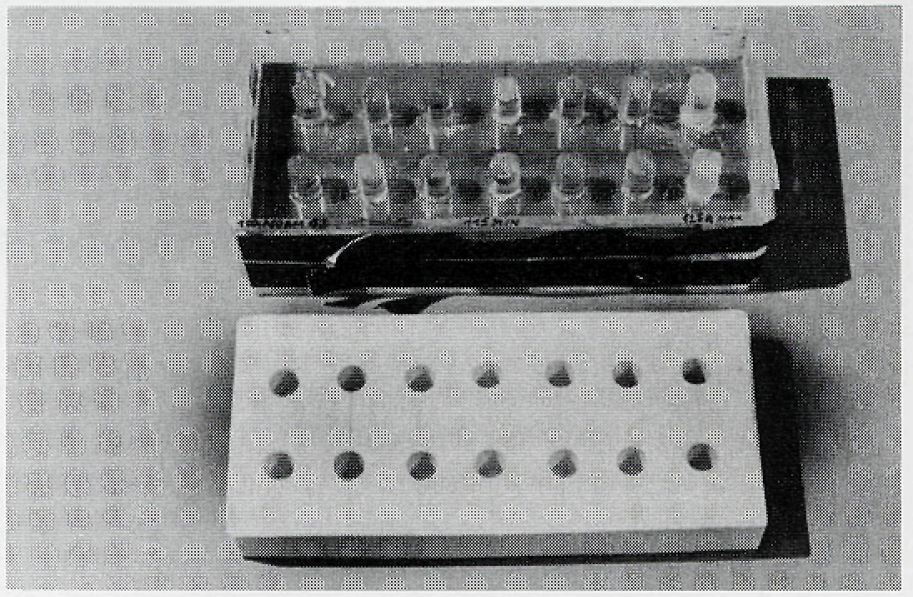

Figure 1B: Assembled primary mold and finished embedding mold for making BEEMcapsule shaped embedding cavities. 


\section{Keeping a good image around the lab just got a lot easier. Quartz PCI.}

With the Windows-based Quartz $\mathrm{PCI}^{\mathrm{TM}}$ system, you can capture and manage highresolution digital images from just about any image-producing device. And do so with a single - and familiar - family of software that lets you smoothly work with SEMs, STEMs, TEMs, light microscopes, video sources, TWAIN devices and CD SEMs. You end continuous learning of new software. Easily network diverse instruments. And conveniently enter all types of images into one database.

Further, after acquiring an image, PCI's processing and display capabilities let you do just about anything you want with it-from annotating, zooming, smoothing and sharpening it to making gamma adjustments and constructing anaglyph stereo images. And you're free to add your own custom functions as well.

Now, think about your database. PCI's scalable family of database solutions ensures your images-plus related documents, custom fields and tables-become part of a powerful database that provides data when,

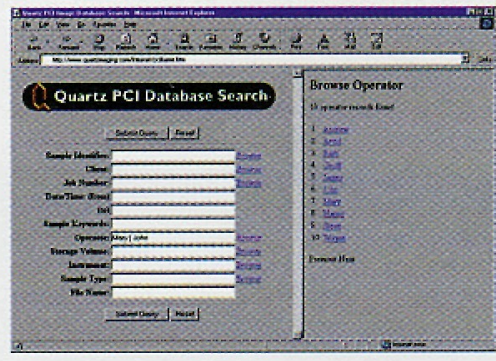

The PCI Intranet Image Server lets you use your Web browser to search for images. where, and in the form needed. OBDC compliance ensures compatibility with third-party tools like Microsoft ${ }^{\Phi}$ Access. ${ }^{\text {TM }}$ And PCI's Workgroup Database, for facilities with up to 25 users, is upgradable to our Enterprise Database which - based on either Oracle ${ }^{\oplus}$ or Microsoft ${ }^{\oplus}$ SQL Server ${ }^{\text {TM }}$ supports thousands. With our Intranet Image Server, you can perform queries and retrieve images via standard Web browsers. And, for off-line storage, there's built-in support for removable media such as CD-ROMs or magneto-optical disks.

So. If easily acquiring, analyzing, manipulating, annotating, transmitting, archiving and retrieving high-resolution images from various sources is in your future, you'll want Quartz PCI details in your hands. You can arrange that easily, too. Visit the Nissei Sangyo Web site for a demonstration, or let us hear from you by phone, FAX or E-mail.

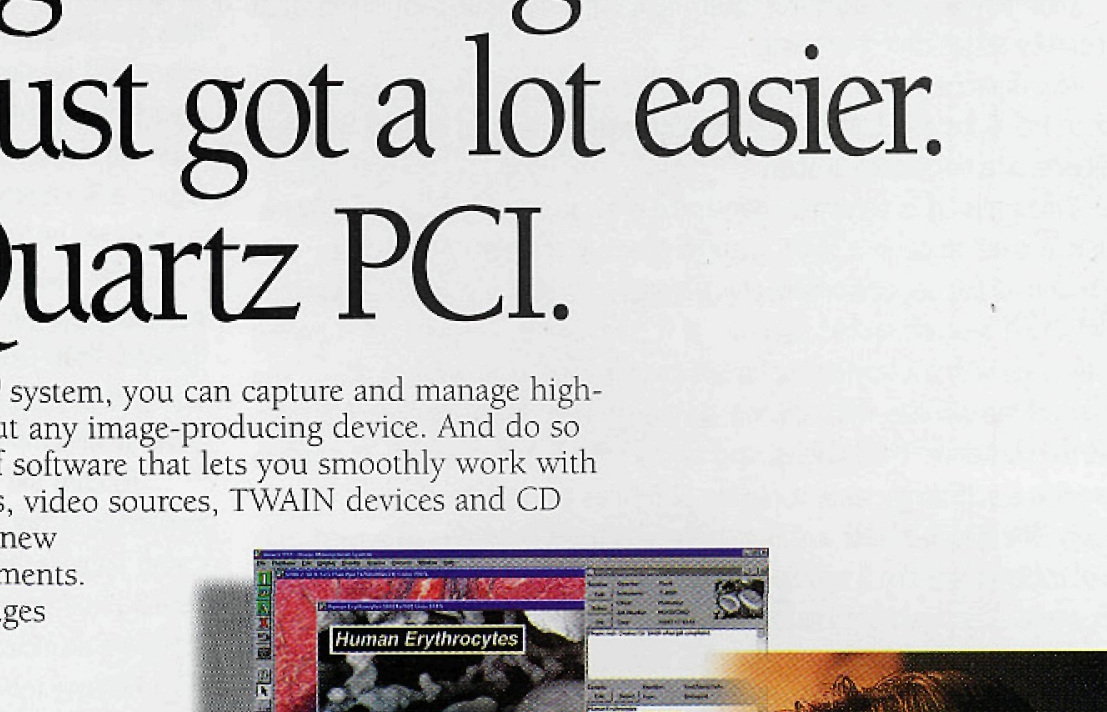




\section{Home Made Silicone Rubber Embedding Molds}

Continued from page 20

Use gloves! The hardener often contains tetra-organo-Zn compounds that can be sensitizing or irritating.

Mix vigorously. The hardener presents an "oily" appearance, and likes to stick to the sides and bottom of the mixing vessel. Be sure that the mixing is complete, and no oiliness is seen.

When mixing is complete, degas the silicone to remove all air bubbles. This is easiest to do in a large vacuum desiccator, empty of desiccant. Line the bottom of the desiccator with paper towels, or spray with a PTFE releasing agent. Don't wipe off spilled silicone, let it cure, and then peel it off. Pumping can be done with a rotary pump, house vacuum, or a hand vacuum pump. The volume of the silicone will increase as it degasses. Admit air before it overflows the container. The bubbles, and so the silicone, will collapse. The trick is to provide a sufficiently large container (2-3 times the volume of mixed silicone rubber). The silicone mass will increase to a point of maximum extension, with lots of bubbles, not like a hydrous solution but more like a very viscous bubble gum mass. After reaching this maximum extension, the silicone mass will collapse back to its pre-evacuation volume. Now it is time to close the valve and admit air (more or less slowly). Don't evacuate any longer since volatile components would be exhausted from the mixture. This could interfere with optimal curing of the mold! If the container is too small to provide the "self collapsing" of the silicone mass, you have to close the valve to stop overflowing the container, then admit air, and then repeat pumping cycles as often as it would be needed to reach the "self collapsing" point of the mass! Proceed as above despite seeing "bubbles" on the surface - they will disappear on pouring.

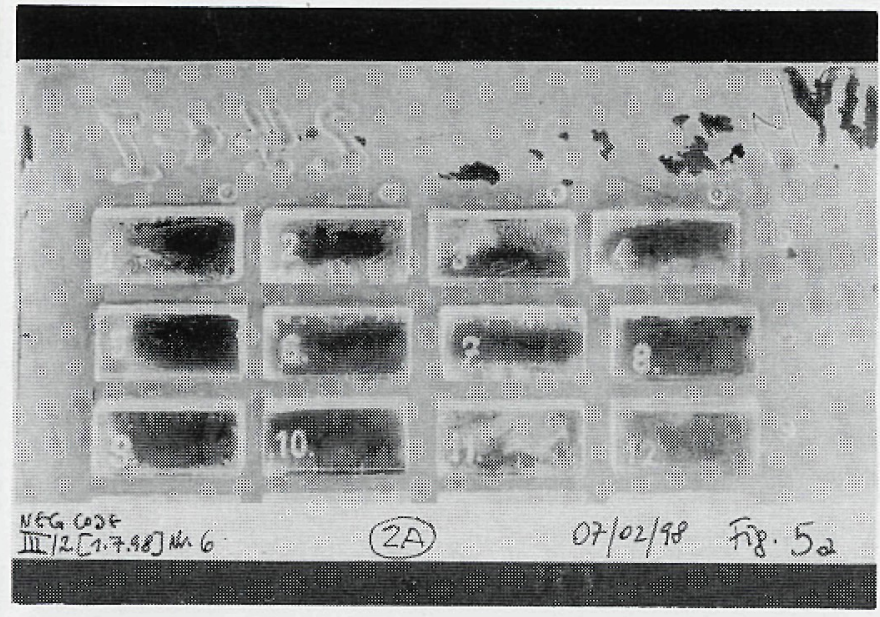

Figure 2A: Detail of slide-in plate for making rectangular embedding cavities, showing specimen block ID numbers engraved in base plate blocks.

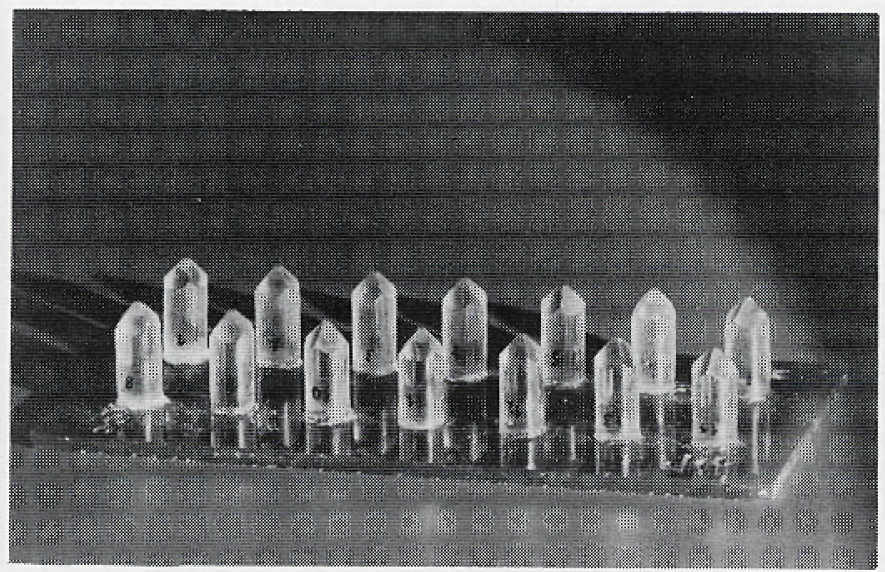

Figure 2B: Slide-in plate used for making BEEM-capsule shaped embedding cavities.
The silicone will be very viscous, but reasonably pourable. A small amoun will remain in the container. If needed to fill the mold, this can be carefully scrapped into the mold. Be very careful during pouring not to introduce air bubbles into the silicone. Start pouring slowly, and speed up towards the end of the pour. Start pouring where there are no cavity forms. After the silicone has covered all of the forms, pouring can be speeded up and moved around the mold. Note: be certain that the silicone has completely spread around the bases and edges of the cavity forms before they are covered by more silicone! Otherwise air bubbles will be trapped at these points, with defective embedding cavities.

After pouring, as a product of initial curing, there will likely be air bubbles in the surface of the mold. These will not affect the embedding cavities and can be ignored. They, except for the larger bubbles, will eventually disappear during curing. If desired, these bubbles can be popped with a needle, or "exploded" with a fine jet of compressed air.

To cure the silicone, let the mold stand covered in a perfectly horizonta position in a dry, temperature-controlled environment for 15 to 20 hours. The curing process may be hastened by heating moderately in an oven at 50 to $100^{\circ} \mathrm{C}$. This usually is not wise when using plexiglass! After this, soft "fluidy" areas are an indication of poor mixing of silicone and hardener.

Remove the polymerized embedding mold from the primary mold by removing the fourth side of the primary mold, then carefully remove the finished silicone embedding mold from the sides. Remove the slide-in plate and peel off the silicone mold from the molding forms of the slide-in plate. If the slide-in plate is stuck to the base plate, carefully separate the silicone from the sides, and peel the silicone form from the slide-in plate by carefully lifting the mold's edges.

Durability of the embedding mold can be improved if, after condensation, curing is tempered at $120^{\circ}$ to $140^{\circ} \mathrm{C}$ for about 12 to 48 hours - after allowing the polymerized mold to sit for one to two days to finish outgassing curing products.

Note: These conditions, especially the time, may vary depending on the silicone rubber used. Also, polymerization may be accelerated in an oven, its temperature depending on the silicone used.

These silicone molds will last for 20 to 30 embedding and de-embedding cycles, and 50 cycles is easily achieved. The following will lengthen lifespans

$\rightarrow$ Do not overfill the embedding cavities with resin. The walls dividing the cavities will be destroyed more quickly with overfilling;

$\rightarrow$ Spray the molds after or before use (at least every tenth or fifteenth use) with a thin layer of releasing agent - but don't spray every time, or there will be remnants of the releasing agent left on the specimen blocks.

Note: Compound names are for products available in Europe. The same, or closely similar, products are available under different names, from perhaps different companies, elsewhere in the world. In North America, try SylGuard from Dow Corning Chemical $\mathrm{Co}$.

\section{Photographs (Micrographs) by Dr. Arno Laminger, Salzburg, Austria}

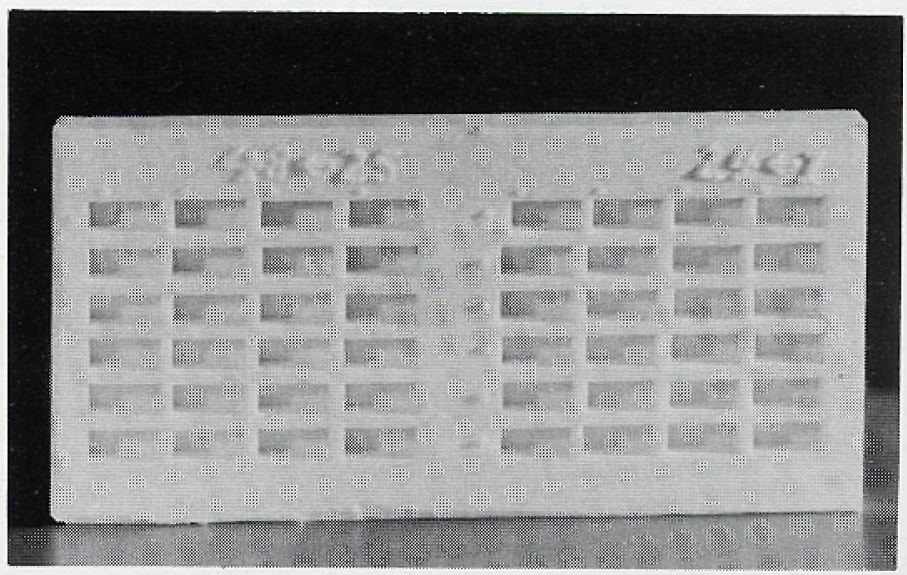

Figure 3: Finished specimen embedding molds showing two possible cavity - numbering schemes 


\section{"A Must" for Cell Biologists... says Trends in Cell Biology}

\section{Cells: A Laboratory Manual}

Edited by David L. Spector, Cold Spring Harbor Laboratory; Robert Goldman, Northwestern University Medical School; Leslie Leinwand, University of Colorado at Boulder

Having identified a gene product, how do you determine what it does? The answer lies in Cells, a new manual designed to do for studies of cell biology what Cold Spring Harbor's Molecular Cloning has done for molecular biology.

- Sets the standard for techniques of proven bench reliability needed by all biomedical scientists studying cellular structure and function

- Delivers consistent, precisely crafted step-by-step protocols in an accessible format, with essential background details and in-depth advice on pitfalls and problem solving

- Created by three distinguished cell biologist/educators, from the contributions of over 180 leading cell biologists

- Complete with more than 300 expertly selected and superbly reproduced illustrations, over 70 in color

- Here's what the reviewers have to say:

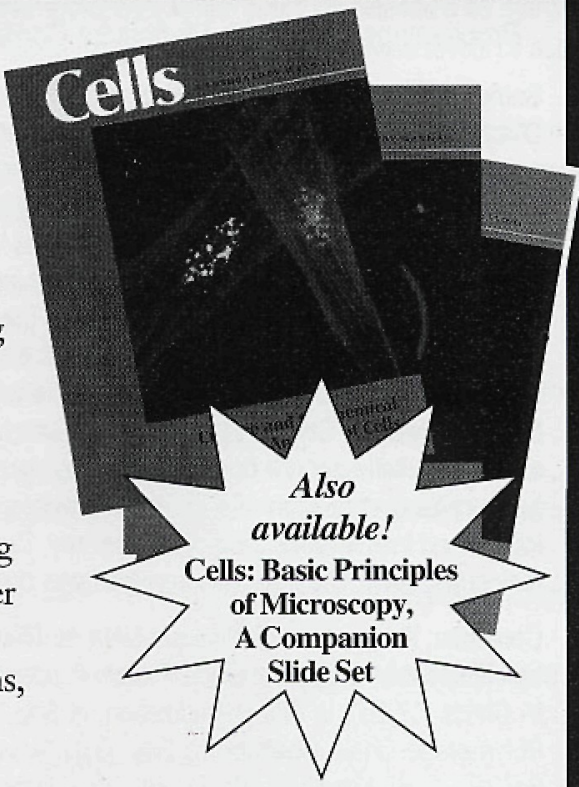

"Cells...an indispensable addition to any modern life science laboratory." - GÜNTER BLOBEL, Rockefeller University

"This is another bible for the modern era of biological researchers, joining the two crucial staples Molecular Cloning and Antibodies....This three volume text fills a gap that has cried for attention, to produce an authoritative and straightforward text that describes the theory and practice of the principal basic-and-beyond techniques for molecular cell biology."

- Trends in Genetics

"This manual is a 'must' for any laboratory planning to use cell-biology techniques. It will be of great use both to beginners and for those who have been chained to the bench a little longer!...so interesting and well written that I and members of the laboratory found ourselves reading sections for methods that we might never consider..."

- Trends in Cell Biology

1998, 2136 pp., illus., color plates, appendices, index

Cloth $\$ 350$ (three-book set)

ISBN 0-87969-522-6

Plastic comb binding $\$ 250$ (three-book set)

Basic Principles of Microsopy Slide Set \$200 (141 slides, 26 pp. booklet)

ISBN 0-87969-521-8

ISBN 0-87969-558-7

\section{CONTENTS}

Volume I Culture and Biochemical Analysis of Cells

Section 1: Cell Culture and Analysis

Section 2: Metabolic Labeling and Protein Modification

Section 3: Subcellular Fractionation

Section 4: Protein Identification and Analysis

Section 5: Protein Expression and Interactions

Section 6: Antibodies as Tools in Cell Biology

Volume II Light Microscopy and Cell Structure

Section 7: Observation of Live Cells and Cellular Dynamics

Section 8: Preparation of Macromolecules and Introduction into Cells

Section 9: Light and Epifluorescence Microscopy

Section 10: Confocal and Multiphoton Microscopy and

Deconvolution
Volume III Subcellular Localization of Genes and their Products Section 11: Visualization of Organelles, Proteins and Gene

Expression

Section 12: In Situ Hybridization

Section 13: Electron Microscopy

Appendices

Appendix 1: Stock Solutions, Buffers, and Media Commonly Used in Cell Biology

Appendix 2: Basic Information for Cell Biologists

Appendix 3: Microscopy: Lenses, Filters, and Emission/Excitation Spectra

Appendix 4: Localization Markers for Subcellular Components

Appendix 5: Cautions

Appendix 6: Suppliers

Index

To order or request additional information:

Call: $\quad$ 1-800-843-4388 (Continental US and Canada) 516-349-1930 (All other locations)

FAX: $\quad 516-349-1946$

E-mail: cshpress@cshl.org or WWW Site http://www.cshl.org/

Write: CSHL Press, 10 Skyline Drive, Plainview, NY 11803-2500 